Pesq. Vet. Bras. 35(Supl.1):56-60, dezembro 2015

DOI: $10.1590 / \mathrm{S} 0100-736 \mathrm{X} 2015001300010$

\title{
Arquitetura comparativa dos pulmões de camundongos normais e afetados pela Distrofia Muscular de Duchenne ${ }^{1}$
}

\author{
Thais B. Lessa ${ }^{2 *}$, Dilayla K. Abreu², Bruno M. Bertassoli³ e Carlos E. Ambrósio ${ }^{4}$
}

\begin{abstract}
Lessa T.B., Abreu D.K., Bertassoli B.M. \& Ambrósio C.E. 2015. [Comparative lung architecture of normal and by Duchenne Muscular Dystrophy affected mice.] Arquitetura comparativa dos pulmões de camundongos normais e afetados pela Distrofia Muscular de Duchenne. Pesquisa Veterinária Brasileira 35(Supl.1):56-60. Departamento de Cirurgia, Programa de Pós-Graduação em Anatomia dos Animais Domésticos e Silvestres, Faculdade de Medicina Veterinária, Universidade de São Paulo, Av. Prof. Dr. Orlando Marques de Paiva 87, São Paulo, SP 05508-270, Brazil. E-mail: thaisblessa32@gmail.com

The Duchenne Muscular Dystrophy (DMD) is a recessive genetic disease characterized by progressive muscle weakness of the pelvic and scapular girdle and progressing to respiratory or heart failure. The $m d x$ mouse is a model widely used for studies. Although they possess a milder phenotype, the morphology and biochemistry of the diaphragm are similar to human DMD. We performed a descriptive anatomical study of the pulmonary parenchyma of five $m d x$ animal models and compared these with the lungs of 5 mice BALB/ C57 (Mus musculus). The findings suggest that the $m d x$ model has morphological features similar to BALB/C57 mice and it must be used with caution in clinical trials which involve the lung.
\end{abstract}

INDEX TERMS: Muscular dystrophy, Duchenne, $m d x$, lung morphology, respiratory anatomy.

RESUMO.- A Distrofia Muscular de Duchenne (DMD) é uma doença genética de caráter recessivo que caracterizada por fraqueza muscular progressiva de cintura pélvica e escapular evoluindo para insuficiência respiratória e, ou cardíaca. 0 camundongo $m d x$ é um modelo amplamente utilizado para estudos da DMD. Apesar do fenótipo destes animais serem mais suave, estes apresentam o principal músculo respiratório, o diafragma com morfologia e bioquímica semelhante à DMD humana, fato este que pode comprometer a função respiratória e consequentemente os pulmões. Foi realizado um estudo anatômico descritivo do parênquima pulmonar dos pulmões de 5 animais modelo $m d x$ comparando estes com os pulmões

\footnotetext{
${ }^{1}$ Recebido em 9 de julho de 2015.

Aceito para publicação em 12 de dezembro de 2015.

${ }^{2}$ Departamento de Cirurgia, Programa de Pós-Graduação em Anatomia dos Animais Domésticos e Silvestres, Faculdade de Medicina Veterinária e Zootencia (FMVZ), Universidade de São Paulo (USP), Av. Prof. Dr. Orlando Marques de Paiva 87, São Paulo, SP 05508 270, Brasil. *Autor para correspondência: thaisblessa32@gmail.com

${ }^{3}$ Departamento de Morfologia, Programa de Pós-Graduação em Biologia Celular, Instituto de Ciências Biológicas, Universidade Federal de Minas Gerais (UFMG), Av. Pres. Antônio Carlos 6627, Pampulha, Belo Horizonte, MG 31270-901, Brasil.

${ }^{4}$ Departamento de Medicina Veterinária, Faculdade de Zootecnia e Engenharia de Alimentos (FZEA), USP, Av. Duque de Caxias Norte 225, Pirassununga, SP 13635-900, Brasil.
}

de 5 camundongos BALB/C57 (Mus musculus). Os pulmões foram analisados macroscopicamente e através de microscopia de luz e eletrônica de varredura. Os achados sugerem que o modelo $m d x$ apresenta morfologia pulmonar semelhante aos camundongos BALB/C57 e que seu uso deve ser cauteloso e criterioso em ensaios clínicos que aborde este órgão.

TERMOS DE INDEXAÇÃO: Distrofia muscular, Duchenne, $m d x$, morfologia pulmonar, anatomia respiratória.

\section{INTRODUÇÃO}

A anatomia descritiva constitui parte dos princípios primordiais da pesquisa, uma vez que esta descreve tecidos biológicos fornecendo dados morfológicos importantes para análises de estudos de afecções que envolvem alterações teciduais e reparo.

A Distrofia Muscular de Duchenne (DMD) é uma doença neuromuscular, de origem hereditária de caráter recessivo, incidindo em meninos em 1 a cada 3500 nascidos. Sua evolução é rápida e severa caracterizada por uma mutação do gene que codifica a proteína $427 \mathrm{kDA}$ chamada distrofina. A distrofina corresponde por cerca de apenas $0,002 \%$ da massa proteica da célula muscular estriada, e está localizada na superfície intracelular do sarcolema e se associa a várias glicoproteínas integrais da membrana, formando 
o complexo glicoproteína-distrofina (CDG) (Berne 2004) o qual é responsável pela permeabilidade da membrana das células musculares (Michele \& Campbell 2003). Portanto a ausência desta proteína, nas células musculares na DMD promove morte gradual das mesmas, a medida que estas são submetidas à tensão pelas contrações musculares, e, por conseguinte, observa-se o aparecimento de fibrose (Bergman et al. 2002).

Dentre os sinais clínicos observa-se a fraqueza muscular progressiva inicial de cintura pélvica e posteriormente de cintura escapular e com a evolução da doença, observa-se o quadro clínico de insuficiência respiratória e/ou cardíaca, a principal causa "mortis" na DMD (Caromano 1999, Kornegay et al. 1999, Strober 2006). Os sinais clínicos respiratórios se exacerbam com o aumento da carga mecânica sustentada a cada movimento respiratório, pela musculatura respiratória já enfraquecida promovendo a redução da complacência pulmonar e da mobilidade da caixa torácica e, por conseguinte hipercapnia, hipoventilação pulmonar, hipoxemia (secundária a hipercapnia) e clearence de secreções (Araujo et al. 2004).

0 modelo murino da DMD, camundongo $m d x$ (x-linked muscular dystrophy mouse), é um modelo amplamente utilizado em pesquisas e avaliações pré-clínicas (Bertassoli et al. 2013, Oliveira et al. 2013, Santos et al. 2013) apresentando facilidade em reprodução, uniformidade genética, economia, conveniência para experimentos em laboratórios. Este é caracterizado por uma total ausência da expressão da distrofina no músculo esquelético, ou seja, se tornando homólogos a DMD humana. Nestes animais, a mutação da distrofina ocorre no exon 23 e a inflamação do músculo esquelético inicia-se com 3 semanas de idade e atinge picos entre 8 e 16 semanas (Lynch et al. 2001, Gosselin et al. 2003). Entretanto estes animais apresentam um fenótipo mais brando da DMD e após períodos de pico de inflamação, os músculos do $m d x$ com exceção do músculo diafragma, voltam a apresentar sua função restaurada. Tal compensação funcional nestes animais tem sido atribuída a expressão de utrofina no sarcolema das células musculares de camundongos adultos, que atua restaurando proteínas do complexo distrofina, protegendo o fenótipo da distrofia muscular no camundongo $m d x$ de forma dose-dependente (Zhou et al. 2008, Huang et al. 2011, Van Putten et al. 2012).

Mesmo apresentando um fenótipo mais suave, estes animais são muito utilizados em estudos científicos devido ao grave comprometimento do diafragma, o que favorece $o$ uso deste modelo no estudo de complicações respiratórias, grande responsável por causa mortis na DMD (Huang et al. 2011, Nelson et al. 2011, Lessa et al. 2012). Entretanto, nos perguntamos se tal ciclo evolucional da doença no camundongo $m d x$ é capaz de afetar e modificar sua morfologia pulmonar.

Considerando que este modelo pré-clínico atualmente, vem sendo utilizado em diversos experimentos que abordam a melhora da função respiratória (Huang et al. 2011, Rafael-Fortney et al. 2011, Van Putten et al. 2012) o estudo da morfologia comparativa histológica e ultraestrutural dos pulmões de $m d x$ por meio da microscopia de luz e ele- trônica de varredura e a comparação com animais BALB/ C57 (Mus musculus) tem o objetivo de aprimorar a interpretação e comparação de dados publicados além de identificar possíveis diferenças entre estes dois modelos.

\section{MATERIAL E MÉTODOS}

Foram utilizados 5 camundongos $m d x$ machos, com idade de 5 meses provenientes do Biotério da Faculdade de Medicina do ABC, 5 camundongos BALB/C57 (Mus musculus) machos, com idade de 5 meses provenientes do Biotério do Departamento de Patologia da FMVZ/USP, protocolo de bioética 2045/2010.

Os animais $m d x$ e controles selecionados foram animais de idade avançada, pois estes já apresentaram picos de inflamação muscular e apresentam o principal músculo respiratório, o diafragma afetado. Segundo Grounds et al. (2008) e Willmann et al. (2011), o músculo diafragma destes animais apresentam bioquímica, morfologia e função semelhantes a DMD em humanos, fato este que favorece o aparecimento de implicações respiratóriase portanto a investigação deste estudo.

Os animais foram eutanasiados por overdose anestésica e os pulmões coletados e fixados em solução de paraformaldeido a $4 \%$ e analisados macroscopicamente.

Para a microscopia de luz os fragmentos foram desidratados em série crescente de etanol (70-100\%) e diafanizados em xilol para posterior inclusão em parafina, onde foram realizados cortes de $5 \mu \mathrm{m}$ em micrótomo (Leica RM 2065) coloração com Hematoxilina-Eosina e Picrossirius Red. As lâminas foram fotomicrografadas em Microscópio Olympus BX 60 acoplado a câmera Axio CAM HRc, utilizando-se o software Zeiss ${ }^{\circledR}$ KS 400.

Para a microscopia eletrônica de varredura (MEV), adaptou-se a metodologia descrita por Lessa et al. 2012 para o processamento dos pulmões. Foram confeccionados blocos e estes cortados em $10 \mu \mathrm{m}$ em micrótomo (Leica RM 2065) para obtenção das lâminas, e posteriormente desparafinizados em estufa a $60^{\circ} \mathrm{C}$ por 2 horas. Em seguida as lâminas foram colocadas por 10 minutos no xilol para eliminação da parafina. Estas foram secas no aparelho de ponto crítico (Balzers CPD020). Na sequência as lâminas foram cobertas com ouro por meio de do processo de "sputtering" (EMITEC H K550) e a observação ultraestrutural e a fotodocumentação realizaram-se em microscópio eletrônico de varredura (LEO 435VP).

\section{RESULTADOS}

Macroscopicamente, observou-se que o pulmão direito de todos os camundongos estudados nesta pesquisa apresentaram, assim como descrito por Fox et al. (2007), uma anatomia peculiar apresentando quatro lobos, o inferior, médio, superior e retrocava, enquanto que o pulmão esquerdo apresentou somente um lobo pulmonar (Fig.1A e Aa).

Na MEV observou-se em todos os camundongos do grupo de $m d x$ um discreto espessamento na parede alveolar quando comparados com o grupo de animais de linhagem BALB/ C57 (Fig.1B e C). Assim como na MEV, microscopicamente observamos um discreto espessamento na parede alveolar no grupo dos camundongos $m d x$, dado este não observado no grupo dos camundongos BALB/C57 (Fig.1D, E e Ee).

$\mathrm{Na}$ análise de Picrossírius sob luz polarizada, não se observou marcação de fibras colágenas em todos os animais BALB/C57 analisados (Fig.2A), entretanto em todos os modelos $m d x$ (Fig.2B) foram observados através da birrefringência do tecido na cor vermelha, fibras colágenas, localizados por todo parênquima pulmonar. 


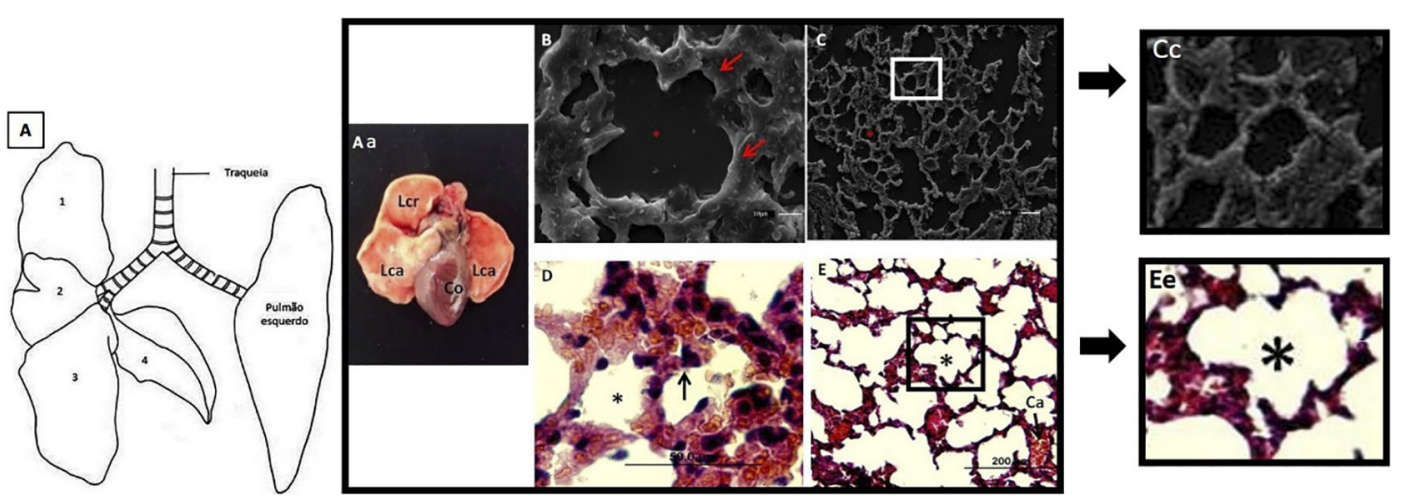

Fig.1. (A) Esquema representativo do pulmão de camundongo: lobo superior (1), lobo médio (2), lobo inferior (3), lobo retrocava (4). (Aa) Pulmão de camundongo $m d x$ : coração (Co), lobo pulmonar cranial (Lcr), lobo pulmonar caudal (Lca). (B, C e Cc) Ampliação da imagem. (C) Imagem de microscopia eletrônica de varredura. (B) Camundongo $m d x$. (C e Cc) Camundongo BALB/C57: alvéolo (*) e setas $(\rightarrow$ ) indicando espessamento da parede alveolar. (Cc) Sem presença de espessamento da parede alveolar. (D, E e Ee) Ampliação da imagem C, parênquima pulmonar. (D) Camundongo mdx. (E e Ee) Camundongo BALB/C57: alvéolo $\left({ }^{*}\right)$. (D) Setas $(\rightarrow)$ indicando espessamento da parede alveolar em camundongo $m d x$. (E) Vaso sanguíneo (Ca).

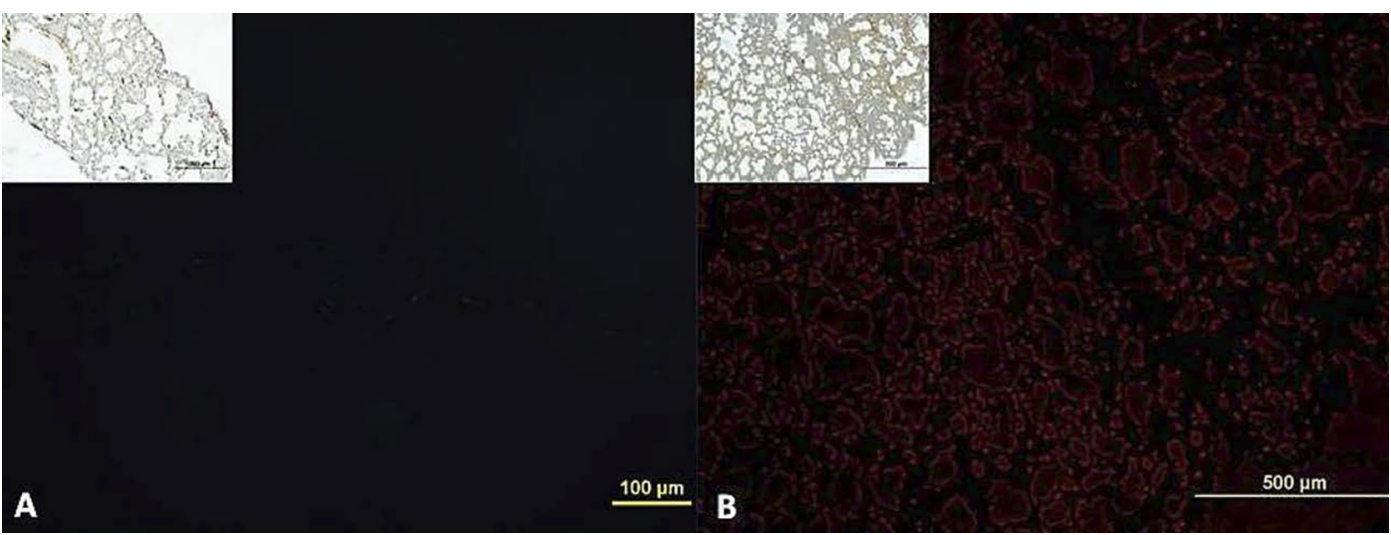

Fig.2. Comparação do parênquima pulmonar entre o camundongo BALB/C57 e $m d x$. Coloração de Picrossírius sob luz polarizada. (A) Parênquima pulmonar de camundongo BALB/C57. (B) Parênquima pulmonar de camundongo $m d x$ com pontos vermelhos, indicando a presença de fibras colágenas.

\section{DISCUSSÃO}

A estrutura e a forma do sistema respiratório podem variar entre as espécies e refletir na demanda de oxigênio (Miller et al. 1993, Ibe et al. 2011), sendo assim a arquitetura pulmonar se correlaciona afetando a capacidade difusora de oxigênio dos alvéolos para os eritrócitos nos capilares pulmonares (Barbier \& Bachofen 2000).

Conforme foi observado o parênquima pulmonar dos camundongos BALB/C57 apresentaram-se como um pulmão típico de mamíferos, sendo constituídos de bronquíolos, alvéolos, capilares sanguíneos circundados por musculatura lisa, corroborando assim com as afirmações de Junqueira \& Carneiro (2004), Plopper \& Adams (2006) e Samuelson (2007), e validando desta forma os achados ultraestruturais deste estudo.

Macroscopicamente o pulmão dos camundongos estudados ( $m d x$ e BALB/C) apresentaram-se conforme descrito por Fox et al. (2007). Na coloração de Picrossírius sob luz polarizada, não observou alteração do parênquima pulmonar.

Nos animais de linhagem $m d x$ a histologia apresentouse alterada com sacos alveolares mais delgados, não se as- semelhando a descrição de Junqueira \& Carneiro (2004) e com os achados dos animais de linhagem BALB/C57. Na ultraestrutura observaram-se as paredes alveolares mais delgadas e os sacos alveolares preservados. Na coloração de Picrossírius Red sob luz polarizada, observaram-se através da birrefringência do tecido pontos na cor vermelha indicando as fibras colágenas, sugerindo áreas de possível formação de fibrose decorrente do processo inflamatório observado na DMD.

Histologicamente as características do parênquima pulmonar descritas por Junqueira \& Carneiro (2004), em humanos e por Santos et al. (2011) em quatis (Nasua nasua), mostram que o parênquima pulmonar se assemelham entre os mamíferos, incluindo os animais da linhagem BALB/ C57 utilizados em nossos estudos e diferentemente dos camundongos $m d x$.

Relacionando os achados histológicos do camundongo $m d x$ com a DMD, podemos observar que o aumento da carga mecânica sustentada a cada movimento respiratório pela musculatura enfraquecida, alterou discretamente a morfologia dos pulmões de $m d x$, sugerindo que no camundongo $m d x$ pode ocorrer a redução da complacência 
pulmonar, hipercapnia, hipoventilação e hipoxemia, entretanto na idade analisada neste estudo, não foi observado alteração significativa da morfologia pulmonar. Em adição a estes achados, notamos pela coloração de Picrossírius a presença de fibras colágenas, indicando a presença de possível fibrose no parênquima pulmonar.

Estudos realizados para avaliar a capacidade e volumes pulmonares na DMD, constataram uma diminuição na capacidade difusora de oxigênio (Caromano et al. 1999, Gozal 2000, Fonseca et al. 2007, Birnkrant et al. 2010). 0 modelo $m d x$, analisado neste estudo, apresentou discretas alterações no parênquima pulmonar, confirmando a correlação descrita entre a arquitetura pulmonar e a capacidade difusora de oxigênio.

Entretanto, não só a arquitetura pulmonar pode influenciar na capacidade difusora de oxigênio e acarretar alterações morfológicas pulmonares, como relata Huang et al. (2000), onde a perda gradual da força muscular respiratória faz com que o paciente com DMD a apresentar diminuição da tosse e da ventilação, pneumonia, atelectasia e insuficiência respiratória.

Ferro et al. (2010), em estudo sobre a atividade da catalase no pulmão, rim e intestino delgado não esquemiado de ratos após perfusão intestinal, constatou pequena congestão vascular, presença de infiltrado polimorfonuclear, acentuada congestão nos septos alveolares e áreas de atelectasias configurando uma inflamação. Móran et al. (2011) em um estudo sobre a inalação de Aspergillus fumigatus em ratos para induzir uma inflamação pulmonar observou nos achados histológicos a presença de severa atelectasia, infiltrado perimorfonuclear, peribronquiolar e alveolar. Tal descrição se assemelha aos achados histológicos encontrados dos animais $m d x$ e difere dos BALB/C57.

\section{CONCLUSÕES}

Diante os dados verificados nesta pesquisa sugerimos que o ciclo evolucional da DMD no camundongo $m d x$ não é capaz de alterar significamente a morfologia pulmonar do camundongo $m d x$ aos 5 meses de idade mesmo, tendo sido observado achados sugestivos de processo inflamatório como espessamento alveolar e congestão dos septos alveolares.

Tal resultado indica que o uso deste modelo deve ser cauteloso e criterioso em relação a experimentos que abordem o pulmão do $m d x$ uma vez que a arquitetura morfológica pulmonar deste modelo aos 5 meses de idade se assemelha com os animais de linhagem BALB/C57.

Agradecimentos.- À FAPESP-Fundação de Amparo a Pesquisa do Estado de São Paulo, pelo apoio financeiro, Processo FAPESP 2012/04506-3.

\section{REFERENCIAS}

Araujo A.P.Q.C., Deco M.C., Klôh B.S., Costa M.R., Góis F.V. \& Guimarães A.F.C.M. 2004. Diagnosis delay of duchenne muscular dystrophy. Revta Bras. Saúde Mater. Infant. 4:179-183.

Barbier A. \& Bachofen H. 2000. The lung of the marmoset (Callithrix jacchus): ultrastructure and morphometric data. Resp. Physiol. 120:167-177.

Bergman R.L., Inzana K.D., Monroe W.E., Shell L.G., Liu L.A., Engvall E. \& Shelton G.D. 2002. Dystrophin-deficient muscular dystrophy in a Labrador Retriever. J. Am. Anim. Hosp. Assoc. 38:255-261.
Berne R.M. 2004. Fisiologia. Elsevier, Rio de Janeiro. 705p.

Bertassoli B.M., Lessa T.B., Santos A.C., Oliveira D.M., Feder D., Ambrósio C.E. \& Assis Neto A.C. 2013. Análise morfológica do estômago de camundos distróficos "mdx". Revta Electrón. Vet. 14:1-9.

Birnkrant D.J., Bushby K.M.D., Amin R.S., Bach J.R., Benditt J.O., Michelle M.E, Finder J.D., Kalra M.S., Kissel J.T., Koumbourlis A.C. \& Kravitz R.M. 2010. The Respiratory Management of Patients With Duchenne Muscular Dystrophy: a DMD care considerations working group specialty article. Ped. Pulmonol. 45:739-748.

Caromano F.A.C. 1999. Características do portador de distrofia muscular de duchenne (DMD). Arq. Ciênc. Saúde Unipar 3:211-218.

Ferro C.C., Chagas V.L.A., Oliveira M.F., Oliveira P.L. \& Schanaider A. 2010. Atividade da catalase no pulmão, rim e intestino delgado não isquemiado de ratos após reperfusão intestinal. Revta. Col. Bras. Cirur. 37:31-38.

Fonseca J.G., Machado M.J.F. \& Ferraz C.L.M.S. 2007. Distrofia muscular de Duchenne: complicações respiratórias e seu tratamento, Revta Ciênc. Med. 16:109-120.

Fox J.G., Barthold S.W. \& Davisson M.T. 2007. The Mouse in Biomedical Research. Elsevier. Cambridge, USA. 791p.

Gosselin L.E., Barkley J.E., Spencer M.J., McCormick K.M. \& Farkas G.A. 2003. Ventilatory dysfunction in $m d x$ mice: impact of tumor necrosis factor-alpha deletion. Muscle and Nerve 28:336-343.

Gozal D. 2000. Pulmonary manifestations of neuromuscular disease with special reference to Duchenne muscular dystrophy and spinal muscular atrophy. Ped. Pulmonol. 29:141-150.

Grounds M.D., Radley H.G., Lynch G.S., Nagaraju K. \& De Luca A. 2008. Towards developing standard operating procedures for pre-clinical testing in the mdx mouse model of Duchenne muscular dystrophy. Neurobiol. Dis. 31:1-19.

Huang P., Cheng G., Lu H., Aronica M., Ransohoff R.M. \& Zhou L. 2011. Impaired respiratory function in $\mathrm{mdx}$ and $\mathrm{mdx} / \mathrm{utrn}(+/-)$ mice. Muscle and Nerve 43:263-267.

Huang P., Zhao X.S., Fields M., Ransohoff R.M. \& Zhou L. 2000. Imatinib attenuates skeletal muscle dystrophy in mdx mice. FASEB J. 23:2539-2548.

Ibe C.S., Salami S.O. \& Onyeanusi B.I. 2011. Macroscopic Anatomy of the Lower Respiratory System in a Nocturnal Burrowing Rodent: African giant pouched rat (Cricetomys gambianus Waterhouse, 1840). Anat. Histol. Embryol. 40:112-119.

Junqueira L.C. \& Carneiro J. 2004. Histologia Básica. Guanabara Koogan, Rio de Janeiro. 487p.

Kornegay J.N., Bogan D.J., Bogan J.R., Childers M.K., Cundiff D.D., Petroski G.F. \& Schueler R.O. 1999. Contraction force generated by tarsal joint flexion and extension in dogs with golden retriever muscular dystrophy. J. Neurol. Sci. 166:115-121.

Lessa T.B., Carvalho R.C., Franciolli A.L., De Oliveira L.J., Barreto R., Feder D., Bressan F.F., Miglino M.A. \& Ambrósio C.E. 2012. Muscle reorganization through local injection of stem cells in the diaphragm of mdx mice. Acta Vet. Scand. 54:1-7.

Lynch G.S., Hinkle R.T. \& Faulkner J.A. 2001. Force and power output of diaphragm muscle strips from $m d x$ and control mice after clenbuterol treatment. Neuromusc. Disor. 11:192-196.

Michele D.E. \& Campbell K.P. 2003. Distrophin-glycoprotein complex: Post- translational processing and dustroglycan function. J. Biol. Chem. 278:15457-15460.

Miller F.J., Mercer R.R. \& Crapo J.D. 1993. Lower Respiratory Tract Structure of Laboratory Animals and Humans: Dosimetry Implications. Aerosol Sci. Technol. 18:257-271.

Móran G., Ojeda G. Diedrichs K., Ortloff A., Barria M. \& Folch H. 2011. Inhalation of Aspergillus fumigatus spores induces airway inflammation in mice in a similar manner as observed in Recurrent Airway Obstruction in horses. Arch. Med. Vet. 43:163-171.

Nelson C.A., Hunter R.B., Quigley L.A., Girgenrath S., Weber W.D., McCullough J.A., Dinardo C.J., Keefe K.A., Ceci L., Clayton N.P., Mcvie-Wylie A., Cheng S.H., Leonard J.P. \& Wentworth B.M. 2011. Inhibiting TGF- $\beta$ activity improves respiratory function in mdx mice. Am. J. Pathol. 178:26112621. 
Oliveira D.M., Santos A.C., Bertassoli B.M., Viana D.C., Prado A.A.F. \& Assis Neto A.C. 2013. Comparative study of the kidneys from dystrofic mice. J. Morphol. Sci. 30:186-190.

Plopper C.G. \& Adams D.R. 2006. Respiratory System, p.153-169. In: Eurell J.A. \& Frappier B.L. (Eds), Textbook of Veterinary Histology. 6th ed. Blackwell Publishing, UK.

Rafael-Fortney J.A., Chimanji N.S., Schill K.E., Martin C.D., Murray J.D., Ganguly R., Stangland J.E., Tran T., Xu Y., Canan B.D., Mays T.A., Delfín D.A., Janssen P.M. \& Raman S.V. 2011. Early treatment with lisinopril and spironolactone preserves cardiac and skeletal muscle in Duchenne muscular dystrophy mice. Circulation 124(5):582-588.

Samuelson D.A. 2007. Sistema respiratório, p.217-240. In: Samuelson D.A. (Ed.), Tratado de Histologia Veterinária. Elsevier, Rio de Janeiro.

Santos A.C., Bertassoli B.M., Oliveira V.C., Souza A.F., Rosa R.A., Carvalho A.F. \& Mançanares C.A.F. 2011. Morfologia da árvore brônquica de quatis (Nasua nasua Linnaeus, 1966). Biotemas 24:75-82.

Santos A.C., Oliveira D.M., Bertassoli B.M., Viana D.C., Vasconcelos B.G. \&
Assis Neto A.C. 2013. Morphology features from mdx mice spleens, used for Ducchene Muscular Dystrophy studies. J. Morphol. Sci. 30:21-27.

Strober J.B. 2006. Therapeutics in duchenne muscular dystrophy. Am. Soc. Exp. Neurotherap. 3:225-234.

Van Putten M., Kumar D., Hulsker M., Hoogaars W.M., Plomp J.J., Van Opstal A., Van Iterson M., Admiraal P., Van Ommen G.J., Hoen P.A. \& Aartsma-Rus A. 2012. Comparison of skeletal muscle pathology and motor function of dystrophin and utrophin deficient mouse strains. Neuromuscul. Disord. 22:406-417.

Willmann R., De Luca A., Benatar M., Grounds M., Dubach J., Raymackers J.M. \& Nagaraju K. 2011. On behalf of the TREAT-NMD Neuromuscular Network. Enhancing translation: guidelines for standard pre-clinical experiments in mdx mice. Neuromuscul. Disor. 22:43-49.

Zhou L., Rafael-Fortney J.A., Huang P., Zhao X.S., Cheng G., Zhou X., Kaminski H.J., Liu L. \& Ransohoff R.M. 2008. Haploinsufficiency of utrophin gene worsens skeletal muscle inflammation and fibrosis in $\mathrm{mdx}$ mice. J. Neurol. Sci. 15:106-111. 